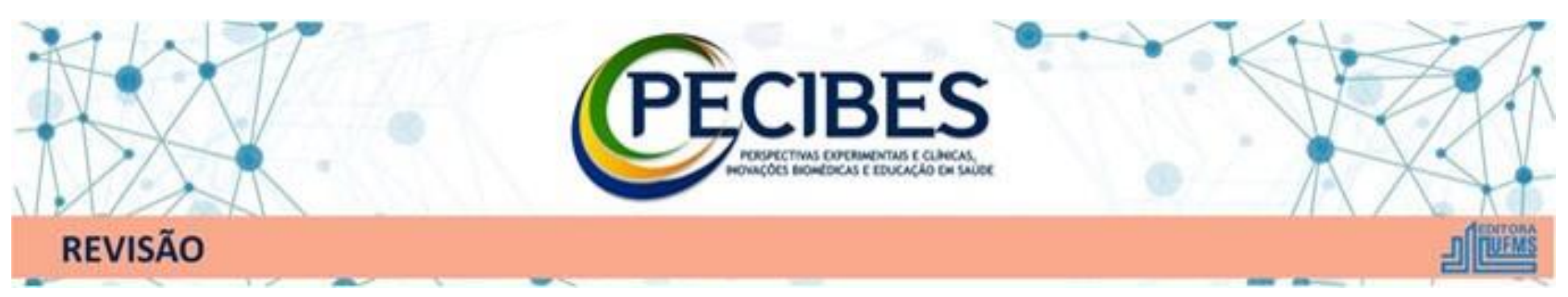

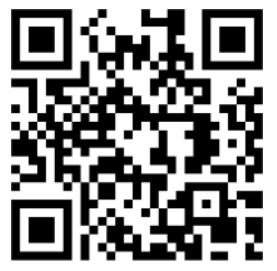

http://www.seer.ufms.br/inde x.php/pecibes/index

*Autor correspondente: Lívia Silveira de Moraes Hilario

E-mail do autor:

livia.moraes@ebserh.go v.br

Palavras-chave: Doença de Parkinson. Antiparkisonianos.

Levodopa (L-DOPA). Corpúsculos de Lewy.

Key-words: Pakinson's Disease.

Antiparkinsonians

Levodoa (L-DOPA).

Lewy bodies.

\section{Descrição da patologia, etiologia e das estratégias farmacológicas e não farmacológicas da Doença de Parkinson}

Description of the pathology, etiology and pharmacocological and non-pharmacocological therapeutic strategies of Parkinson's Disease

\section{Lívia Silveira de Moraes Hilario ${ }^{1}$, Willyan Franco Hilario ${ }^{2}$}

${ }^{1}$ Farmacêutica-Bioquímica da Empresa Brasileira de Serviços Hospitalares (EBSERH) lotada no Hospital das Clínicas da Universidade Federal de Minas Gerais (HC-UFMG), Mestre em Bioquímica e Farmacologia pela Universidade Federal do Espírito Santo (UFES)

${ }^{2}$ Farmacêutico da Empresa Brasileira de Serviços Hospitalares (EBSERH) lotado no Hospital das Clínicas da Universidade Federal de Minas Gerais (HC-UFMG), Mestre em Bioquímica e Farmacologia pela Universidade Federal do Espírito Santo (UFES)

Resumo

A doença de Parkinson (DP) atinge cerca de 6 milhões de pessoas em todo o mundo, sendo a segunda doença neurodegenerativa mais comum. Terapias baseadas na reposição de dopamina são até hoje a principal estratégia de tratamento farmacológico, tendo como a principal droga utilizada a levodopa (L-DOPA). Outras classes de medicamentos são também utilizadas, isoladamente ou em adição a LDOPA, dependendo da idade do paciente, da sintomatologia presente e do grau da doença. Nesta revisão, são descritos os princípios da patologia, etiologia e as principais linhas de tratamento farmacológico da DP, utilizando como fonte de pesquisa artigos publicados nas bases de dados Medline, Scielo e PubMed de estudos clínicos, básicos e de revisão de revistas com reconhecido impacto no meio acadêmico e científico. Apesar de ainda não possuir cura e acometer vários outros neurotransmissores pelo processo da doença, pacientes acometidos pela DP conseguem obter grande benefício com o tratamento sintomático farmacológico, com impacto direto na qualidade de vida.

\section{Abstract}

Parkinson's disease (PD) affects about 6 million people worldwide, being the second most common neurodegenerative disease. Therapies based on dopamine replacement are still the principal pharmacological treatment strategy with levodopa (L-DOPA) as the main drug used. Other classes of drugs are also used, alone or in addition to L-DOPA, depending of the patient age, symptomatology presence and disease's degree. In this review, the pathology, etiology and the of currently pharmacological treatment of PD are described, using as a research source articles published in the Medline, Scielo and PubMed databases of clinical, basic and review studies of journals with recognized impact on academic and scientific environment. Although it still has no cure and affects several other neurotransmitters due to the disease process, patients affected by PD are able to obtain great benefit from symptomatic pharmacological treatment, with direct impact on quality of life. 


\section{Introdução}

A Doença de Parkinson (DP) é uma doença neurodegenerativa, de caráter crônico e progressivo, que tem se tornado cada vez mais frequente devido ao aumento da expectativa de vida da população. Fatores genéticos e ambientais influenciam no risco para o desenvolvimento da DP. Sua patogênese tem sido atribuída à formação de espécies reativas de oxigênio, que induzem o estresse oxidativo, causando a morte dos neurônios dopaminérgicos da substância negra pars compacta $(\mathrm{SNpc})$. Esta região é relacionada ao controle motor, por isso, sintomas motores, como instabilidade postural, tremor de repouso, rigidez muscular, desequilíbrio e bradicinesia, são observados em pacientes com a doença

Sintomas não motores, como alterações do olfato, perda cognitiva, depressão e distúrbios do sono também acometem tais pacientes, visto que neurodegenerações além da SNpc estão presentes.

A principal linha de tratamento, utilizada desde a década de 1960, é baseada na administração de levodopa (LDOPA), que tem como objetivo a reposição da dopamina (DA) perdida no cérebro devido à neurodegeneração, levando à atenuação principalmente dos sintomas motores. Contudo, o efeito da L-DOPA parece ser menor em pacientes com uso prolongado e em estágios mais avançados da doença, possivelmente pelo baixo número de neurônios capazes de metabolizar a L-DOPA a DA nesta fase.

Além da L-DOPA, diversas classes de medicamentos têm sido utilizadas para o tratamento da DP, como os agonistas dopaminérgicos, que atuam diretamente nos receptores de DA; os inibidores da monoamino oxidase (MAO); os inibidores da catecol-O-metiltransferase (COMT); os anticolinérgicos e os antiglutamatérgicos. Não obstante, nenhum destes medicamentos tem mostrado prevenir ou frear o avanço da doença.

Muitos pesquisadores têm estudado substâncias com potencial neuroprotetor, principalmente de origem natural, com propriedades antioxidantes e antiinflamatórias, para o tratamento da DP. Porém, não foi encontrada ainda uma terapia neuroprotetora eficaz para as doenças neurodegenerativas, embora pesquisas experimentais mostrem resultados promissores.

O objetivo deste trabalho de revisão é descrever os princípios patológicos, etiológicos e as principais linhas de tratamento da DP. Adicionalmente, o trabalho visa contribuir para o aperfeiçoamento dos profissionais da área da saúde que lidam diretamente com o tema, tanto no âmbito hospitalar quanto ambulatorial, a fim de melhorar a qualidade de vida dos pacientes acometidos pela DP.

\section{Material e Métodos}

A pesquisa bibliográfica foi realizada nas bases de dados Medline, Scielo e PubMed de estudos clínicos, básicos e de revisão publicados em revistas com reconhecido impacto no meio acadêmico e científico. A pesquisa restringiu-se a trabalhos publicados com a busca de palavras-chaves: "Doença de Parkinson", "Tratamento", "terapia farmacológica" e "antiparkinsonianos".

\section{Resultados}

A DP é uma síndrome neurodegenerativa progressiva que se caracteriza pelo tremor de repouso, rigidez muscular, instabilidade postural, desequilíbrio, acinesia e/ou hipocinesia (BLOEM; OKUN, 2021). Dados epidemiológicos pontuam que a DP é a segunda doença neurodegenerativa mais comum no mundo entre as pessoas idosas e tem prevalência de 2\% (BARTELS; LEENDERS, 2009).

A primeira descrição foi feita pelo médico britânico James Parkinson, numa monografia clássica intitulada como "Um ensaio sobre a paralisia trepidante", de 1817, na qual a descreveu como uma "paralisia agitante" (BLOEM; OKUN, 2021). Jean Charcot, posteriormente, adicionou mais informações à descrição inicial do quadro clínico, como a micrografia (caligrafia com letras pequenas que se reduzem progressivamente), presença de alteração postural, rigidez muscular, bradicinesia e ainda sugeriu a mudança do nome da enfermidade para Doença de Parkinson, em homenagem ao autor da primeira descrição. Além disso, Charcot sugeriu a presença de comprometimento da memória e disfunções cognitivas nos pacientes com DP (RICHARDSON et al., 2018).

A gênese neuroquímica da DP foi desvendada por Ehringer e Hornykiewicz, em 1960 e republicado em 1998, os quais mostraram que a concentração de DA da SNpc e do núcleo estriado era extremamente pequena em encéfalos postmortem de pacientes parkinsonianos. Concordando com esses dados, a observação da despigmentação da SNpc foi associada à morte de neurônios dopaminérgicos da via nigroestriatal (Figura 1) (BLOEM; OKUN, 2021; DAUER; PRZEDBORSKI, 2003). Uma segunda característica, porém fisiopatológica e menos específica, é a presença de inclusões eosinofílicas citoplasmáticas denominadas corpúsculos de Lewy em diversas regiões mesencefálicas (Figura 1) (LEE; LIU, 2008).

O comprometimento dos níveis de DA e da transmissão dopaminérgica são os principais responsáveis pelos sintomas motores observados na doença. Esse comprometimento influencia diretamente a atividade da via nigroestriatal e dos componentes responsáveis pelo controle da atividade motora, os gânglios da base. (ARMSTRONG; OKUN, 2020).

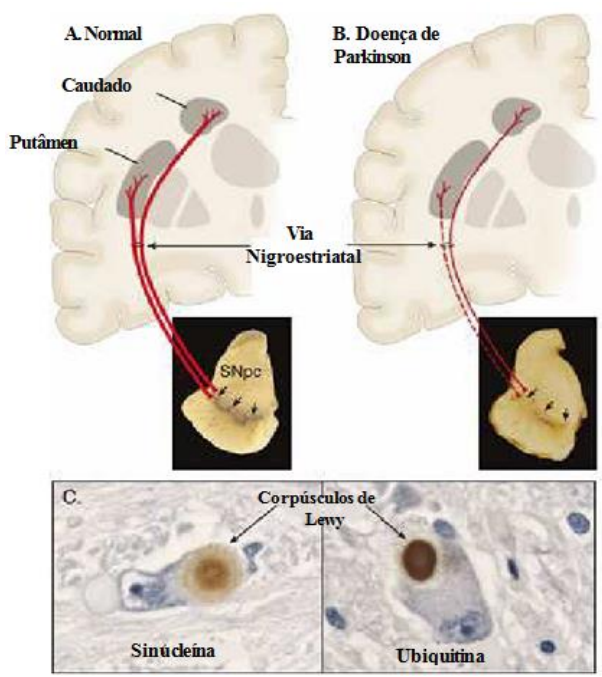


Figura 1: Esquema simplificado da via nigroestriatal normal (A) e na DP (B) mostrando a despigmentação da SNpc e consequente degeneração da via nigroestriatal, e em (C) imunohistoquímica mostrando os chamados corpúsculos de Lewy em neurônios dopaminérgicos na SNpc. Adaptado de: DAUER; PRZEDORSKI (2003).

Embora a DP seja definida e caracterizada como uma doença motora, hoje, sabe-se que pacientes com DP apresentam declínio e outras alterações cognitivas e psiquiátricas. $\mathrm{O}$ declínio cognitivo tem sido descrito frequentemente nos estágios mais precoces da doença, antes mesmo dos sintomas motores, sendo que, com o curso da doença a magnitude deste aumenta. Tais alterações são representadas especialmente pelo comprometimento da memória, foco atencional e tomada de decisão e da flexibilidade cognitiva (MORIGUCHI; YABUKI; FUKUNAGA, 2012). Além disso, o declínio cognitivo relacionado ao desenvolvimento de depressão associada à DP tem sido relatado com frequência nesses pacientes (BOGGIO et al, 2006; FREGNI et al, 2004).

Em um estudo clínico realizado num grande hospital da Dinamarca, Pfeiffer e colaboradores (2013), descreveram e caracterizaram o perfil cognitivo de pacientes com DP em estágios precoces. O estudo, que contou com 86 pacientes com idades de 40 a 70 anos, revelou que $69 \%$ dos pacientes apresentaram comprometimento de memória, 54\% disfunções executivas e $35 \%$ déficits de atenção. Além disso, o prejuízo cognitivo observado foi relacionado a um maior nível de bradicinesia, rigidez postural e simetria axial consoante à escala unificada da DP (UPDRS - Unified Parkinson Disease Rating).

Os mecanismos que conduzem ao processo neurodegenerativo na DP ainda são obscuros, apesar de muitas evidências sugerirem que a neurodegeneração seja um processo multifatorial (REALE et al, 2009). Estudos básicos e clínicos realizados nas últimas décadas têm revelado muito sobre a patogênese da DP. As principais hipóteses aceitam que o estresse oxidativo, o envelhecimento, a neuroinflamação, a condição genética do indivíduo e a exposição a certas condições ambientais contribuem para o desenvolvimento da doença (DAUER; PRZEDBORSKI, 2003).

O estado de desarmonia entre a formação e a neutralização de espécies reativas de oxigênio (ROS) é definido como estresse oxidativo. Tal condição é uma das causas do dano neuronal observado em inúmeras doenças neurológicas incluindo a DP (MOSLEY et al, 2006). Regiões encefálicas ricas em catecolaminas, como a SNpc, podem ser mais vulneráveis na geração de ROS (MOSLEY et al, 2006).

A metabolização da DA favorece a produção de peróxido de hidrogênio $\left(\mathrm{H}_{2} \mathrm{O}_{2}\right)$, ânion superóxido $\left(\mathrm{O}_{2-}\right)$ e DAquinona. Adicionalmente, esses neurônios da SNpc possuem menor reserva mitocondrial em relação a outras populações neuronais, o que torna esta região do mesencéfalo particularmente mais suscetível aos efeitos nocivos das ROS (ARMSTRONG; OKUN, 2020; BLOEM; OKUN, 2021). Níveis elevados de $\mathrm{H}_{2} \mathrm{O}_{2}$ podem reagir com o grupo heme de certas proteínas, como a hemoglobina e o citocromo, gerando o radical hidroxila $\left(\mathrm{HO}^{-}\right)$, o qual é capaz de oxidar carboidratos, lipídios, proteínas e o ácido desoxirribonucleico
(DNA) (BARBOSA et al, 2006; MOSLEY et al, 2006). Adicionalmente, $\mathrm{O}_{2}^{-}$e a DA-quinona podem reagir com resíduos proteicos de cisteína, danificando várias proteínas celulares (DAUER; PRZEDBORSKI, 2003).

A neurodegeneração da SNpc observada na DP tem sido correlacionada também com o envelhecimento e o aumento da expressão e da atividade da MAO (HILARIO et al 2016). Evidências mostram que o aumento da atividade da MAO ativa determinadas vias bioquímicas que facilitam a formação de ROS e exacerbam a metabolização da DA desencadeando uma maior formação de DA-quinona a qual interrompe a cadeira respiratória (DAUER; PRZEDBORSKI, 2003).

No contexto da neuroinflamação, tem sido demonstrado que a formação e a presença dos corpúsculos de Lewy facilitam a ação neurotóxica do sistema imune inato mediada pelas células da micróglia (MOSLEY et al, 2006). O aumento da atividade dessas células, que é persistente em pacientes com DP, libera inúmeros fatores neuroinflamatórios como, por exemplo, o fator maturador da glia (GMF) o qual desencadeia o aumento de expressão do fator de necrose tumoral alfa (TNF- $\alpha$ ), interleucina 1 beta (IL-1 $\beta$ ) e da cicloxigenase-2 (COX-2) e das prostaglandinas (PGs) (KHAN et al, 2015). Portanto, os processos inflamatórios associados ao aumento de expressão do TNF- $\alpha$, IL-1 $\beta$, COX2 e às altas concentrações de PGs estão envolvidos em eventos deletérios que promovem a neurodegeneração da SNpc (KHAN et al, 2015).

É importante ressaltar que cerca de 10 a $20 \%$ dos pacientes com DP apresentam um histórico familiar da doença o qual pode ser tanto autossômico dominante como autossômico recessivo (BLOEM; OKUN, 2021). As alterações genéticas relacionadas afetam principalmente os genes de codificação da $\alpha$-sinucleína, parkina e componentes do sistema proteassômico (BLOEM; OKUN, 2021; LEE; LIU, 2008).

Adicionalmente, um amplo número de evidências mostra que o contato e a exposição a agentes ambientais específicos e até certos hábitos podem influenciar e acarretar o processo neurodegenerativo em indivíduos geneticamente susceptíveis. A exposição a metais pesados, toxinas, inseticidas, pesticidas e herbicidas tem se destacado como um importante fator associado ao aumento do risco de desenvolvimento da DP nessa população (BLOEM; OKUN, 2021; PARKINSON STUDY GROUP, 1993).

Apesar da DP ainda não ter cura, os medicamentos atualmente utilizados são focados na melhora dos sinais e sintomas motores e não motores, proporcionando mais qualidade de vida aos portadores da doença. $\mathrm{O}$ tratamento sintomático é recomendado para os pacientes desde o momento do diagnóstico, devendo ser escolhido o medicamento mais adequado considerando fatores como estágio da doença, sintomatologia presente, ocorrência de efeitos colaterais, idade do paciente, medicamentos em uso e seu custo (ARMSTRONG; OKUN, 2020; BRASIL, 2017). A maioria dos indivíduos com DP usam medicamentos de diferentes classes na tentativa de se obter o melhor efeito terapêutico utilizando doses mais baixas possíveis de levodopa, com os menores efeitos colaterais (BLOEM; OKUN, 2021). As terapias medicamentosas disponíveis para 
a DP estão listadas na tabela 1.

\begin{tabular}{|ll|}
\hline Classe & Drogas \\
\hline Medicamentos dopaminérgicos & Levodopa \\
Inibidores de descarboxilase & Carbidopa \\
& Benserazida \\
Agonistas dopaminérgicos & Bromocriptina \\
& Pramipexol \\
Inibidores da MAO & Selegilina \\
& Rasagilina \\
Inibidores da COMT & Tolcapona \\
& Entacapona \\
Anticolinérgicos & Biperideno \\
& Triexifenidil \\
Antiglutamatérgicos & Amantadina \\
\hline
\end{tabular}

Tabela 1. Manual de recomendações da Academia Brasileira de Neurologia em Parkinson (2010).

A L-DOPA é precursora do neurotransmissor DA, e é a droga mais utilizada e também a mais potente no tratamento da DP (MOREIRA et al., 2007). Após sua absorção intestinal, ela atravessa a barreira hematoencefálica, onde, por ação da enzima dopa descarboxilase (DDC), é convertida em DA pelos neurônios dopaminérgicos remanescentes, pelos neurônios não dopaminérgicos ou pelas células da glia (FERRAZ, 2004). A DA assim produzida é responsável pela eficácia terapêutica do fármaco na DP.

Contudo, o uso prolongado da L-DOPA frequentemente leva a complicações motoras, que são as discinesias e as flutuações motoras, que ocorrem devido às variações de sua concentração sanguínea, necessitando de administrações com intervalos cada vez menores da droga em adição a doses cada vez maiores (ARMSTRONG; OKUN, 2020). Além disso, a L-DOPA é amplamente transformada em DA perifericamente, podendo também causar efeitos colaterais como taquicardia, náuseas, depressão e insônia (FERRAZ, 2004; POEWE, 2006; FLOEL et al., 2008).

Os inibidores de DDC são drogas que inibem a síntese de dopamina. Nas apresentações comerciais de L-DOPA, a combinação com drogas inibidoras da DDC, como a carbidopa e a benzerasida, está sempre presente. Essas preparações diminuem os efeitos colaterais observados com a administração isolada de L-DOPA, pois como a carbidopa e a benzerasida não ultrapassam a barreira hematoencefálica, elas inibem a conversão periférica da L-DOPA à DA, reduzindo, assim, a dose necessária da L-DOPA e aumentando a sua biodisponibilidade (FERRAZ, 2004).

Os agonistas dopaminérgicos podem ser utilizados em qualquer fase da DP (FERRAZ, 2004). Há duas classes de agonistas dopaminérgicos, os ergolínicos e os não ergolínicos. Dentre os disponíveis no Brasil encontram-se a bromocriptina (derivada do ergot) e o pramipexol (não-ergolínico). Para se tentar diminuir os sintomas e retardar o início do uso de levodopa, eles têm sido usados na fase inicial da doença. $\mathrm{Na}$ fase avançada, quando são mais frequentes as discinesias e flutuações motoras, eles são utilizados em associação à LDOPA, pois possuem uma meia vida superior a esta, permitindo uma redução na dose de L-DOPA necessária e melhor controle dos sintomas motores (BRASIL, 2017).

Atualmente, o uso dos agonistas ergolínicos vem diminuindo, em parte pela presença de efeitos colaterais importantes e em outra parte pela introdução dos agonistas dopaminérgicos não-ergolínicos, como o pramipexol e o ropinirol (DIAS-TOSTA et al., 2010). Em 2002, foram descobertos os efeitos nocivos da pergolida (ergolínico) às válvulas cardíacas, o que fez a Food and Drug Administration (FDA) retirar o medicamento do mercado nos Estados Unidos. Apesar de não constar no Protocolo Clínico e Diretrizes Terapêuticas para a DP, é um medicamento ainda utilizado no Brasil. Assim, segundo a Academia Brasileira de Neurologia (DIAS-TOSTA et al., 2010), pacientes que ainda utilizam estas drogas ergolínicas devem ser monitorados rotineiramente quanto à presença de fibrose valvar cardíaca.

As drogas inibidoras da MAO, uma das enzimas responsáveis pela degradação intraneuronal da DA, são utilizadas para aumentar a biodisponibilidade da L-DOPA, desempenhando importante função na presença de flutuações motoras.

A seleginina inibe a MAO-B de forma seletiva e irreversível. Isto torna esta droga livre dos efeitos colaterais que os inibidores da MAO não-seletivos possuem. Ela sofre metabolização hepática, que gera um metabólito de ação semelhante à da anfetamina, causando estimulação catecolaminérgica, que também poderia ter alguma atividade antiparkinsoniana (DIAS-TOSTA et al., 2010). Há evidências de que a sua administração nas fases iniciais da doença podem postergar o uso de L-DOPA (PARKINSON STUDY GROUP, 1993). Não há evidências para a utilização de seleginina nas fases avançadas da DP. Apesar de inicialmente ter se acreditado que tal droga possuísse efeito neuroprotetor devido aos resultados dos ensaios pré-clínicos, estudos clínicos não conseguiram confirmar a esperada neuroproteção (BOLL et al., 2011).

A rasagilina possui características farmacológicas similares à selegilina. Ela foi incluída no Protocolo Clínico e Diretrizes Terapêuticas para a DP em 2017 (BRASIL, 2017) após ter sido demonstrada sua eficácia (HAUSER et al., 2016). Porém, contrariamente à selegilina, a rasagilina não é metabolizada a anfetamina, e sua associação à L-DOPA demonstrou ter melhor controle das flutuações motoras da DP (LEES, 1995; CAI et al., 2015).

Há dois inibidores da COMT, enzima metabolizadora de catecolaminas, a tolcapona e a entacapona. Eles são sempre utilizados em combinação com a L-DOPA, por não possuírem efeito antiparkinsoniano isoladamente. A entacapona está presente em comprimidos formulados em conjunto com L-DOPA e carbidopa, fazendo com que os níveis plasmáticos de L-DOPA sejam superiores e mais sustentados do que após administração de L-DOPA e carbidopa sozinhos, resultando em maior eficácia no controle das flutuações motoras (LIAO et al., 2020)

Os anticolinérgicos foram por muito tempo a única alternativa terapêutica para a DP (FERRAZ, 2004). Acreditase que sua atuação ocorra no desequilíbrio entre DA e a atividade colinérgica estabelecida pela DP. Para indivíduos com tremor proeminente refratário à L-DOPA ou agonistas dopaminérgicos, agentes anticolinérgicos podem ser utilizados, como o biperideno e triexifenidil. Contudo é necessário ter cautela para os efeitos adversos, particularmente os relacionados à cognição, dado o risco 
aumentado do desenvolvimento de demência devido ao seu uso (RICHARDSON et al., 2018). Portanto, medicações anticolinérgicas podem ser utilizadas no tratamento sintomático da DP, tanto em monoterapia quanto de forma combinada, mas não devem ser a droga de primeira escolha (DIAS-TOSTA et al., 2010).

Discinesias são tratadas através da redução na administração de drogas dopaminérgicas ou por adição de amantadina. O mecanismo de ação da amantadina no tratamento da DP ainda não é bem definido, mas sabe-se que é um antagonista não seletivo e não competitivo de baixa sensibilidade do receptor N-metil-d-aspartato com propriedades antiglutamatérgicas e também com algum controle sobre a liberação da dopamina (LIAO et al., 2020; MORAES et al., 2016; MIZOGUCHI et al., 1994). Devido ao seu efeito limitado e de curta duração, a amantadina pode ser usada como monoterapia para tratamento da fase inicial da $\mathrm{DP}$, porém não como droga de primeira escolha. Em um recente ensaio clínico de fase 3 (LIAO et al., 2020; PAHWA et al., 2017) foi testada uma nova formulação de amantadina de liberação prolongada, sendo verificado diminuição da discinesia induzida por L-DOPA.

Diante das limitações da terapia farmacológica convencional, um grande número de terapias não farmacológicas experimentais têm emergido nas ultimas décadas com resultados promissores para o tratamento $\mathrm{e}$ preveção da DP. Por exemplo, a estimulação transcraniana magnética (TMS) e a estimulação transcraniana por corrente contínua de baixa intensidade (tDCS), quando aplicadas repetidas vezes sobre o córtex pré-frontal dorso lateral esquerdo, se mostraram eficazes na recuperação motora e cognitiva de pacientes com DP e depressão associada (MORAES et al., 2016; BOGGIO PS et al, 2006; FREGNI et al, 2004). Ducan e Earhart (2012), utilizando outra ferramenta não farmacológica num estudo clínico, relataram melhora motora em pacientes idosos com DP que iniciaram a prática de Tango. Esse achado reforça a ideia de que o exercício físico voluntário podepor proteger e reverter alguns sintomas característicos da DP (FREDRIKSSON et al, 2011; GERECKE et al, 2010). Em 2016 nosso grupo de pesquisa demonstrou, em estudos independentes, que o extrato etanólico de Combretum Leprossum, uma planta da flora brasileira, e a exposição a um ambiente enriquecido, um paradigma que envolve a manipulação ambiental com estimulos cognitivos e sensoriais, são capazes de prevenir o comprometimento motor e as anormalidades bioquímicas e moleculares dos sistemas dopaminérgico e colinérgico num modelo murino da DP (MORAES et al., 2016; HILARIO et al., 2016). Portanto, o estudo, a identificação e a melhor compreensão dos principais mecanismos e alvos relacionados a influência dessas terapias experimentais pode fornecer as bases para o desenvolvimento racional de novos fármacos e justificar práticas de atividades físicas, sociais e culturais como forma de prevenção da DP e até de outras doenças neurodegenerativas (NITHIANANTHARAJAH; HANNAN, 2006; PANG; HANNAN, 2013).

\section{Conclusão}

A DP há muito tempo é vista como um distúrbio causado pela degeneração do sistema dopaminérgico, levando às manifestações motoras clássicas da doença. No entanto, agora existem evidências esmagadoras de que a DP é mais do que uma doença meramente causada pela deficiência da dopamina. É sabido que uma infinidade de outros neurotransmissores é afetada pelo processo da doença.

Duzentos anos após a publicação "Essay on the Shaking Palsy", na qual o médico James Parkinson descreveu pela primeira vez a doença que ficou conhecida pelo seu nome, ainda não foi descoberta uma terapia neuroprotetora que fosse capaz de prevenir ou curar a doença. Assim, recomenda-se que o tratamento farmacológico deve ser iniciado, preferencialmente, quando do diagnóstico da DP.

O melhor esquema terapêutico para a DP, atualmente, é aquele que permite o melhor controle motor com os menores efeitos adversos, devendo ser levado em consideração fatores como estágio da doença, sintomatologia presente, ocorrência de efeitos colaterais, idade do paciente, medicamentos em uso e seu custo.

\section{Declaração}

Os autores declaram que não existe conflito de interesse.

\section{Referências}

Armstrong MJ, Okun MS. Diagnosis and Treatment of Parkinson Disease: A Review. Journal of the American Medical Association, 323, 6, 548-560, 2020.

Barbosa MT, et al. Parkinsonism and Parkinson's disease in the elderly: a community-based survey in Brazil (the Bambui study). Movement Disorders, 21(6):800-8. 2006.

Bartel A, Leenders KL. Parkinson's disease: the syndrome, the pathogenesis and pathophysiology. Cortex, 45, 8, 915-21, 2009.

Benbir $G$ et al. Hospital-based study: risk factors in development of motor complications in 555 Parkinson's patients on levodopa therapy. Clinical neurology and neurosurgery, 108, 8, 726-32, 2006.

Bloem BR, Okun MS. Parkinson's disease. Thee Lancet. 397, 10297, 2284-2303, 2021.

Boggio PS, et al. Effects of transcranial direct current stimulation on working memory in patients with Parkinson's disease. Journal of the neurological sciences, 249, 1, 31-38, 2006.

Boll M C. et al. Medical Management of Parkinson's Disease: Focus on Neuroprotection. Current Neuropharmacology, 9, 2, 350- 9, 2011.

Brasil, Ministério da Saúde (MS). Portaria Conjunta $n^{\circ} 10$ de 31 de outubro de 2017. Aprova o Protocolo 
Clínico e Diretrizes Terapêuticas - Doença de Parkinson. Diário Oficial da União 2017; 09 out.

Cai JP, et al. Safety and efficacy of rasagiline in addition to levodopa for the treatment of idiopathic Parkinson's disease: a meta-analysis of randomised controlled trials. European Neurology, 73, 1-2, 5$12,2015$.

Dauer W, Przedborski, S. Parkinson's Disease: Mechanisms and Models. Neuron, 39, 6, 889-909, 2003.

Dias-Tosta E, et al. Doença de Parkinson-recomendações. Academia Brasileira de Neurologia, $1^{a}$ ed., 2010.

Ducan R, Earhart, G. Randomized controlled trial of community-based dancing to modify disease progression in Parkinson disease. Neurorehabilitation and neural repair, 26, 2, 13243, 2012.

Ferraz HB. Agonistas Dopaminérgicos no tratamento da Doença de Parkinson. Revista Neurociências, 12 4, 2004.

Floel A, et al. Levodopa increases memory encoding and dopamine release in the striatum in the elderly. Neurobiology of Aging, 2, 267-79, 2008.

Fredriksson et al. Running wheel activity restores MPTPinduced functional deficits. Journal of neural transmission, 118, 3, 407-20, 2011.

Fregni F, et al. Repetitive transcranial magnetic stimulation is as effective as fluoxetine in the treatment of depression in 75 patients with Parkinson's disease. Journal of neurology, neurosurgery, and psychiatry, 75, 8, 1171-74, 2004.

GBD 2016 Parkinson's Disease Collaborators. Global, regional, and national burden of Parkinson's disease, 1990-2016: a systematic analysis for the Global Burden of Disease Study 2016. Lancet Neurology, 7, 11, 939-953, 2018.

Gerecke, et al. Exercise protects against MPTP-induced neurotoxicity in mice. Brain research, 1341, 7283, 2010.

Hauser RA, et al. Efficacy of rasagiline in early Parkinson's disease: a metaanalysis of data from the TEMPO and ADAGIO studies. International Journal of Neuroscience, 126, 10, 942-6, 2016.

Hilario WF, et al. Cholinergic and Dopaminergic Alterations in Nigrostriatal Neurons Are Involved in Environmental Enrichment Motor Protection in a Mouse Model of Parkinson's Disease. Journal of Molecular Neuroscience, 60, 4, 453-464, 2016.

Khan M M, et al. Absence of Glia Maturation Factor
Protects Dopaminergic Neurons and Improves Motor Behavior in Mouse Model of Parkinsonism. Neurochemical Research, 40, 5, 980-90, 2015.

Lee F, Liu F. Genetic factors involved in the pathogenesis of Parkinson's disease. Brain Research Reviews, 58, 2, 354-64, 2008.

Lees A, Comparison of therapeutic effects and mortality data of levodopa and levodopa combined with selegiline in patients with early, mild Parkinson's disease. Parkinson's Disease Research Group of the United Kingdom. BMJ, 311, 1602-1607, 1995.

Liao $X$, et al. Levodopa/carbidopa/entacapone for the treatment of early Parkinson's disease: a metaanalysis. Neurol Sci, Disponível em: https://doi.org/10.1007/s10072-020-04303-x, 2020. Acesso em 29 de nov. 2021.

Mizoguchi K, et al. Amantadine increases the extracellular dopamine levels in the striatum by re-uptake inhibition and by N-methyl-Daspartate antagonism. Brain Research, 662, 255-258, 1994.

Moraes LS, et al. Medicinal plant Combretum leprosum mart ameliorates motor, biochemical and molecular alterations in a Parkinson's disease model induced by MPTP. Journal of Ethnopharmacology. 185, 68-76, 2016.

Moreira CS, et al. Doença de Parkinson: como diagnosticar e tratar. Revista Científica da Faculdade de Medicina de Campos, Rio de Janeiro, 2, 2, 19-29, 2007.

Moreira CS, et al. Doença de Parkinson: como diagnosticar e tratar. Revista Científica da FMC, Vol. 2, 2, 2007.

Moriguchi S, Yabuki Y, Fukunaga K. Reduced calcium/calmodulindependent protein kinase II activity in the hippocampus is associated with impaired 79 cognitive function in MPTP-treated mice. Journal of neurochemistry, 120, 4, 541-51, 2012.

Mosley L, et al. Neuroinflammation, oxidative stress, and the pathogenesis of Parkinson's disease. Clinical Neuroscience Research, 6, 1, 261-81, 2006.

Nithianantharajah J, Hannan A J. Enriched environments, experience-dependent plasticity and disorders of the nervous system. Nature reviews. Neuroscience, 7, 9, 697-709, 2006.

Pahwa R, et al. ADS-5102 (Amantadine) ExtendedRelease Capsules for Levodopa-Induced Dyskinesia in Parkinson Disease (EASE LID Study): A Randomized Clinical Trial. Journal of the American Medical Association, 74, 8, 941-949, 2017. 
Pang T, Hannan A. Enhancement of cognitive function in models of brain disease through environmental enrichment and physical activity. Neuropharmacology, 64, 515-28, 2013.

Parkinson J, et al. An Essay on the Shaking Palsy. The Journal of Neuropsychiatry and Clinical Neurosciences, 14, 2, 223-236, 2002.

Pfeiffer H C. et al. Cognitive impairment in early-stage non-demented Parkinson's disease patients. Acta neurologica Scandinavica,129, 5 307-18, 2013.

Reale M, et al. Peripheral cytokines profile in Parkinson's disease. Brain, behavior, and immunity, 23, 1, 5563, 2009.

Robakis D, Fahn S. Defining the Role of the Monoamine Oxidase-B Inhibitors for Parkinson's Disease. CNS Drugs, 29, 6,433-441, 2015.

Yabuki Y, et al. Nobiletin treatment improves motor and cognitive deficits seen in MPTP-induced Parkinson model mice. Neuroscience, 259, 12641, 2014. 